\title{
Reducing the risk of infection in a patient who will undergo splenectomy
}

\author{
Dan Petrescu MD, Wayne L. Gold MD, Jerome A. Leis MD
}

\begin{abstract}
A 28-year-old woman with steroid-refractory immune thrombocytopenia (platelet count $16 \times$ $10 \%$, with associated epistaxis) recently saw her hematologist, who recommended elective splenectomy for the management of her condition. ${ }^{1}$ After a discussion with her hematologist, the patient has ongoing concern regarding her risk of infection following this procedure, and she presents to her primary care physician to discuss strategies to reduce this risk.
\end{abstract}

\section{What is this patient's risk of infection after splenectomy?}

Elective splenectomy is indicated in the management of certain medical conditions (Table 1). ${ }^{1,2}$ Patients who have undergone splenectomy are at risk of overwhelming postsplenectomy infection, which is characterized by sepsis, meningitis or both, and carries a fatality rate of $50 \%-70 \%$. $^{3}$ These episodes occur in patients who have had splenectomy at a rate of $0.2 \%-0.5 \%$ per year, with a lifetime risk of about 5\%. Streptococcus pneumoniae is the most common pathogen ( $>50 \%$ ), followed by other encapsulated bacteria such as Haemophilus influenzae and Neisseria meningitidis, and less commonly by gramnegative organisms such as Escherichia coli, and species of Salmonella and Pseudomonas. ${ }^{4}$

\section{What immunizations are recommended before and after elective splenectomy?}

To reduce the risk of overwhelming postsplenectomy infection and other invasive bacterial infections, vaccinations should be administered at least two weeks before elective splenectomy. Canadian immunization guidelines recommend the 23-valent pneumococcal polysaccharide vaccine (Pneu-P-23), the conjugate $H$. influenzae type $\mathrm{B}$ vaccine, and both the meningococcal $\mathrm{C}$ conjugate (Men-C-C) and polysaccharide (MenP-ACYW135) vaccines. ${ }^{5}$ More durable protection against serogroup $\mathrm{C}$ meningococcal disease may be achieved by giving Men-C-C two weeks before vaccination with Men-P-ACYW135. In a retrospective population-based cohort study, seasonal influenza immunization reduced the incidence of death in this patient population from 61.7 cases per 1000 person-years to 27 cases per 1000 person-years (hazard ratio $0.46,95 \%$ confidence interval 0.33-0.62) and is therefore recommended annually. ${ }^{2.5}$ Booster vaccinations are recommended for adults five years after the initial vaccination with both Men-P-ACYW135 and Pneu-P-23, and continuing every five years thereafter with Men-P-ACYW135. ${ }^{5}$ Complete recommendations are outlined in the 2006 Canadian Immunization Guide. ${ }^{5}$

\section{What investigations and management are appropriate if fever develops post- splenectomy?}

Fever in a patient who has undergone splenectomy is a medical emergency and requires prompt assessment and management. For all patients presenting with fever after splenectomy, blood cultures should be done. A lumbar puncture should be performed if meningitis is suspected. Expert opinion recommends that empiric antibiotic therapy includes an intravenous thirdgeneration cephalosporin (e.g., ceftriaxone or cefotaxime), combined with vancomycin to ensure coverage of drug-resistant pneumococci. ${ }^{3}$ Despite a lack of clinical evidence, some experts also recommend a "pill-in-pocket" approach with patient self-administration of amoxicillin or

Table 1: Most common indications for elective splenectomy in a Canadian population-based study ${ }^{2}$

Medical condition

Splenectomies/ 1000 person-years

\begin{tabular}{|ll|}
\hline Immune thrombocytopenia & 4.04 \\
\hline Hemolytic anemia & 3.63 \\
\hline Non-Hodgkin lymphoma & 2.84 \\
\hline Hodgkin lymphoma & 1.32 \\
\hline Hypersplenism & 0.40 \\
\hline
\end{tabular}
declared.

This article has been peer reviewed.

Correspondence to:

Dr. Jerome A. Leis, jerome.leis@utoronto.ca

CMAJ 2012. DOI:10.1503 /cmaj.120159
Competing interests: None 
amoxicillin-clavulanate at the onset of febrile illness and instructions to the patient to present for urgent medical assessment. ${ }^{6}$ Lifelong antibiotic prophylaxis in adults is controversial; however, daily penicillin has been suggested for patients with ongoing immune or hematologic disease. ${ }^{6}$

\section{What other advice can be offered to reduce the patient's risk of postsplenectomy infection?}

Patient awareness and education is paramount in preventing infections after splenectomy. A cohort study has shown that patients with the most knowledge about their condition had a prevalence of overwhelming postsplenectomy infection of $1.4 \%$ compared with $16.5 \%$ among patients with little knowledge $(p<0.001){ }^{7}$ Experts suggest routine use of medical alert bracelets and laminated information cards by patients with asplenia. ${ }^{6}$ Patients should also be educated on the risks of contracting fatal infections from animal bites (e.g., Capnocytophaga canimorsus from dogs) or from exposure during travel (e.g., malaria, babesiosis), and about

Box 1: Strategies to reduce the risk of infection in a patient who will undergo splenectomy

- Vaccinations are indicated before and after splenectomy to reduce the risk of overwhelming postsplenectomy infection.

- Yearly influenza vaccination has been shown to reduce mortality.

- Patients who have undergone splenectomy should be educated to seek prompt medical assessment in case of fever, animal bites or travel-related illness.

- Laminated patient information cards and medical alert bracelets are suggested for all asplenic patients. the need for prompt medical assessment in these situations. ${ }^{6}$

\section{The case revisited}

Following her visit with her primary care physician, the patient had a better understanding of her risk of infection postsplenectomy and the interventions that could be used to reduce this risk (Box 1). She received the indicated vaccinations before and after her splenectomy; she now wears a medical alert bracelet notifying health care providers of her asplenic state. Three years after her procedure, her immune thrombocytopenia remains in remission (platelet count $175 \times 10^{9} / \mathrm{L}$ ). She receives annual influenza vaccination, which she did not receive before her splenectomy.

\section{References}

1. Neunert C, Lim W, Crowther M, et al.; American Society of Hematology. The American Society of Hematology 2011 evidence-based practice guideline for immune thrombocytopenia. Blood 2011;117:4190-207.

2. Langley JM, Dodds L, Fell D, et al. Pneumococcal and influenza immunization in asplenic persons: a retrospective populationbased cohort study 1990-2002. BMC Infect Dis 2010;10:219.

3. Davidson RN, Wall RA. Prevention and management of infections in patients without a spleen. Clin Microbiol Infect 2001;7: 657-60.

4. Di Sabatino A, Carsetti R, Corazza GR. Post-splenectomy and hyposplenic states. Lancet 2011;378:86-97.

5. National Advisory Committee on Immunization. Canadian immunization guide. 7th ed. Ottawa (ON): The Public Health Agency of Canada; 2006. p. 119-121.

6. Spelman D, Buttery J, Daley A, et al. Guidelines for the prevention of sepsis in asplenic and hyposplenic patients. Intern Med J 2008;38:349-56.

7. El-Alfy MS, El-Sayed MH. Overwhelming postsplenectomy infection: Is quality of patient knowledge enough for prevention? Hematol J 2004;5:77-80.

Affiliations: From the Department of Medicine (Petrescu), University of Toronto, and the Division of Infectious Diseases (Gold, Leis), University Health Network, Toronto, Ont.

Contributors: Wayne Gold and Jerome Leis were involved in the conception of the article. Dan Petrescu performed the literature review and drafted the report. All authors revised the article and approved the final version to be published.

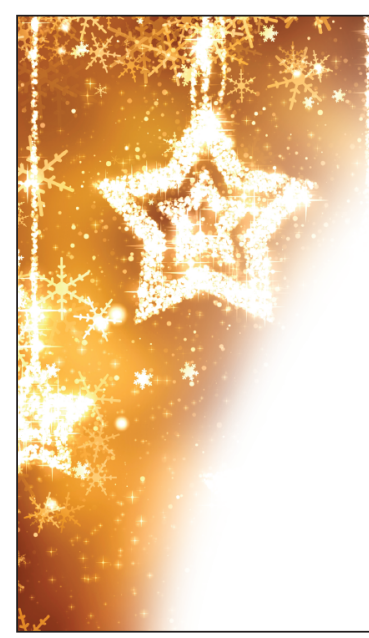

\section{Call for papers: CMAJ Holiday Reading}

Hilarity and good humour ... help enormously in both the study and the practice of medicine. - William Osler

Submit your twisted research, biting satire or culturally sensitive missives to CMAJ's award-winning, intensely cited Holiday Reading section. Articles should be no longer than 1200 words; photographs and illustrations are most welcome. Submit your article online at http://mc.manuscriptcentral.com/cmaj. If you need more information, kindly contact barbara.sibbald@cmaj.ca

Deadline: October 1, 2012 